\title{
A high-definition 3D exoscope as an alternative to the operating microscope in spinal microsurgery
}

\author{
Sebastian Siller, MD, Caroline Zoellner, Manuel Fuetsch, MD, Raimund Trabold, MD, \\ Joerg-Christian Tonn, MD, and Stefan Zausinger, MD
}

\begin{abstract}
Neurosurgical Clinic, Clinic of the University of Munich (LMU), Campus Grosshadern, Munich, Germany
OBJECTIVE Since the 1970s, the operating microscope (OM) has been a standard for visualization and illumination of the surgical field in spinal microsurgery. However, due to its limitations (e.g., size, costliness, and the limited movability of the binocular lenses, in addition to discomfort experienced by surgeons due to the posture required), there are efforts to replace the OM with exoscopic video telescopes. The authors evaluated the feasibility of a new 3D exoscope as an alternative to the $\mathrm{OM}$ in spine surgeries.
\end{abstract}

METHODS Patients with degenerative pathologies scheduled for single-level lumbar or cervical spinal surgery with use of a high-definition 3D exoscope were enrolled in a prospective cohort study between January 2019 and September 2019. Age-, sex-, body mass index-, and procedure-matched patients surgically treated with the assistance of the OM served as the control group. Operative baseline and postoperative outcome parameters were assessed. Periprocedural handling, visualization, and illumination by the exoscope, as well as surgeons' comfort level in terms of posture, were scored using a questionnaire.

RESULTS A 3D exoscope was used in 40 patients undergoing lumbar posterior decompression (LPD) and 20 patients undergoing anterior cervical discectomy and fusion (ACDF); an equal number of controls in whom an OM was used were studied. Compared with controls, there were no significant differences for mean operative time (ACDF: 132 vs 116 minutes; LPD: 112 vs 113 minutes) and blood loss (ACDF: 97 vs 93 ml; LPD: 109 vs $55 \mathrm{ml}$ ) as well as postoperative improvement of symptoms (ACDF/Neck Disability Index: $p=0.43$; LPD/Oswestry Disability Index: $p=0.76$ ). No intraoperative complications occurred in either group. According to the attending surgeon, the intraoperative handling of instruments was rated to be comparable to that of the OM, while the comfort level of the surgeon's posture intraoperatively (especially during "undercutting" procedures) was rated as superior. In cases of ACDF procedures and long approaches, depth perception, image quality, and illumination were rated as inferior when compared with the OM. By contrast, for operating room nursing staff participating in 3D exoscope procedures, the visualization of intraoperative process flow and surgical situs was rated to be superior to the OM, especially for ACDF procedures.

CONCLUSIONS A 3D exoscope seems to be a safe alternative for common spinal procedures with the unique advantage of excellent comfort for the surgical team, but the drawback is the still slightly inferior visualization/illumination quality compared with the OM.

https://thejns.org/doi/abs/10.3171/2020.4.SPINE20374

KEYWORDS exoscope; video telescope; 3D; 4K; operating microscope; spine surgery; microsurgery; surgical technique

$\mathrm{I}$ N 1962, Jacobson et al. ${ }^{1}$ were the first to report the use of an operating microscope $(\mathrm{OM})$ in microsurgery, and in 1977, Caspar $^{2}$ and Yaşargil ${ }^{3}$ were the first to implement the OM in spine surgery. The OM has since become a standard for intraoperative visualization in modern microsurgery due to its excellent magnification and illumination of spinal anatomy and pathologies.
The OM enables the placement of microsurgical instruments into the operative field, as well as a wide range of infinitely variable magnification. Its binocular viewing lenses and its hydraulic counterbalance system allow for a $3 \mathrm{D}$ visualization in nearly any direction. However, the OM is expensive and, due to its heavy counterbalance system for stabilization of the head stage, is burdened by impaired

ABBREVIATIONS ACDF = anterior cervical discectomy and fusion; BMI = body mass index; LPD = lumbar posterior decompression; NDI = Neck Disability Index; ODI = Oswestry Disability Index; $\mathrm{OM}=$ operating microscope; $\mathrm{OR}$ = operating room; $\mathrm{PROM}=$ patient-reported outcome measure.

SUBMITTED March 17, 2020. ACCEPTED April 22, 2020.

INCLUDE WHEN CITING Published online July 10, 2020; DOI: 10.3171/2020.4.SPINE20374. 
maneuverability and limited space and movability in the operating room (OR). In addition, the superior 3D visualization is primarily restricted to the operating and assisting surgeons, who may experience intraoperative fatigue because visualization via the OM often forces the surgical team to work in nonergonomic positions.

This face-to-machine interface began to change in the last decade with the development of imaging exoscopes (i.e., exoscopic video telescopes) consisting of a 3D camera, dedicated 3D glasses, and 3D screens with high-definition (or $4 \mathrm{~K}$ ) resolution. The move of surgical imaging from a through-the-lens strategy to a sight-line strategy via a digital screen fundamentally impacts multiple aspects of spinal microsurgery, including OR setup, surgical ergonomics, and software integration. Currently, a variety of exoscopic systems are available for neurosurgical use: the VITOM 3D (KARL STORZ SE \& Co. KG), KINEVO (Carl Zeiss AG), Modus V (Synaptive Medical), and ORBYE (Olympus). Particularly for the VITOM 3D system, several small case series have shown its feasibility, ${ }^{4-10}$ and a recent editorial review of exoscope platforms suggests that the VITOM 3D is well suited for spine procedures. ${ }^{11}$ Apart from these reports, systematic studies comparing the VITOM 3D or other exoscopes with the OM in terms of unique advantages and disadvantages in spine surgeries are still missing.

The purpose of the present study was therefore to systematically evaluate the VITOM 3D exoscope as an alternative to the $\mathrm{OM}$ in spine surgeries with regard to handling, image quality, illumination, and comfort for the entire surgical staff.

\section{Methods \\ Study Design}

Between January 2019 and September 2019, 40 patients who underwent lumbar posterior decompression (LPD) and 20 patients who underwent anterior cervical discectomy and fusion (ACDF) were prospectively enrolled and operated on with the assistance of the VITOM 3D model TH200. The surgical team and OR nurses were educated in how to handle the VITOM 3D prior to the start of the study; surgeons, in particular, underwent additional training in the intraoperative use of the VITOM 3D during a 2 -week period to avoid a bias in the learning curve for study-relevant procedures.

Local institutional review board approval and individual patient consent were obtained prior to study enrollment. For a systematic evaluation, only patients scheduled for a single-level procedure due to degenerative changes (spinal canal stenosis, intervertebral disc herniation) were included, and patients matched for procedure, age, sex, and body mass index (BMI) operated on with use of the OM (M525 F40; Leica Microsystems GmbH) served as the control group. Both 3D exoscope- and OM-based procedures were performed by the same team of experienced surgeons (S.Z., R.T., and M.F.), assisted by one of a pool of 5 spine residents and one of a pool of 5 OR nurses from the neurosurgical department.

After each procedure, the surgeon and assistant responded to a standardized questionnaire regarding the intraoperative handling and positioning of the exoscope and the control unit for magnification/focal length, the handling of the microsurgical instruments, intraoperative depth perception, image quality, illumination, and the level of comfort with regard to intraprocedural posture. The OR nursing staff was asked about handling of the sterile covering and storage of the exoscope in the OR as well as the ability to follow the consecutive procedural steps and appropriately assist the surgeons. Each of the aforementioned aspects could be scored as follows, based on a scoring system adopted from the one previously published by Oertel and Burkhardt: ${ }^{5}$ grade 1 is considered to be excellent (superior to the $\mathrm{OM}$ ), grade 2 is considered to be very good (equal to the OM), grade 3 is considered to be good (almost equal to the OM with minor differences), grade 4 is considered to be satisfactory (considerably inferior to the OM but the surgical team is still able to perform the procedure), and grade 5 is considered to be unsatisfactory (unacceptably inferior to the OM such that conversion to the $\mathrm{OM}$ was necessary). The intraoperative wearing of polarization glasses was scored as grade 1 (noticeable), grade 2 (not disrupting), and grade 3 (disrupting). Additional commentary not addressed by the questionnaire could also be made.

\section{Patient and Procedural Characteristics}

Overall patient characteristics, operative records, complications, and reoperation rates were recorded. The visual analog scale for neck/back and radicular pain was used for evaluation of pain; the Neck Disability Index (NDI) ${ }^{12}$ and Oswestry Disability Index (ODI) ${ }^{13}$ were used to evaluate pain-related disability; and the Barthel Index was used to assess general performance ${ }^{14}$ in daily living in all patients before and after surgery as well as at the 3-month outpatient follow-up visit. Scores for NDI were categorized into grade 1 (no disability: $0 \%-8 \%$ ), grade 2 (mild disability: $10 \%-28 \%$ ), grade 3 (moderate disability: $30 \%-$ $48 \%$ ), grade 4 (severe disability: 50\%-68\%), and grade 5 (complete disability: $>68 \%$ ), while scores for ODI were categorized into grade 1 (minimal disability: 0\%-20\%), grade 2 (moderate disability: 21\%-40\%), grade 3 (severe disability: $41 \%-60 \%$ ), grade 4 (crippling back pain: $61 \%$ $80 \%$ ), and grade 5 (complete disability: $>80 \%$ ) for statistical evaluation. The patients' postoperative general clinical outcome was graded using Odom's criteria. ${ }^{15}$ Postoperatively new or worsened sensorimotor deficits and bladder/ bowel dysfunction were recorded. The patient-reported outcome measures (PROMs) were collected by a senior medical student (C.Z.) specially trained in patient assessment.

\section{Exoscopic Equipment and Intraoperative Setup}

The IMAGE1 S CONNECT module, the IMAGE1 S D3 LINK module, the POWER LED 300 SCB light source, and the light cable constitute the essential basic hardware. The VITOM 3D model TH200 $90^{\circ}$ exoscope (Fig. 1) includes a 4K sensor system with a focal distance of 200-500 mm, an infinitely variable zoom, and a magnification range from approximately $\times 8$ to $\times 30$. For intraoperatively facilitated control of the zoom, focus, region of 


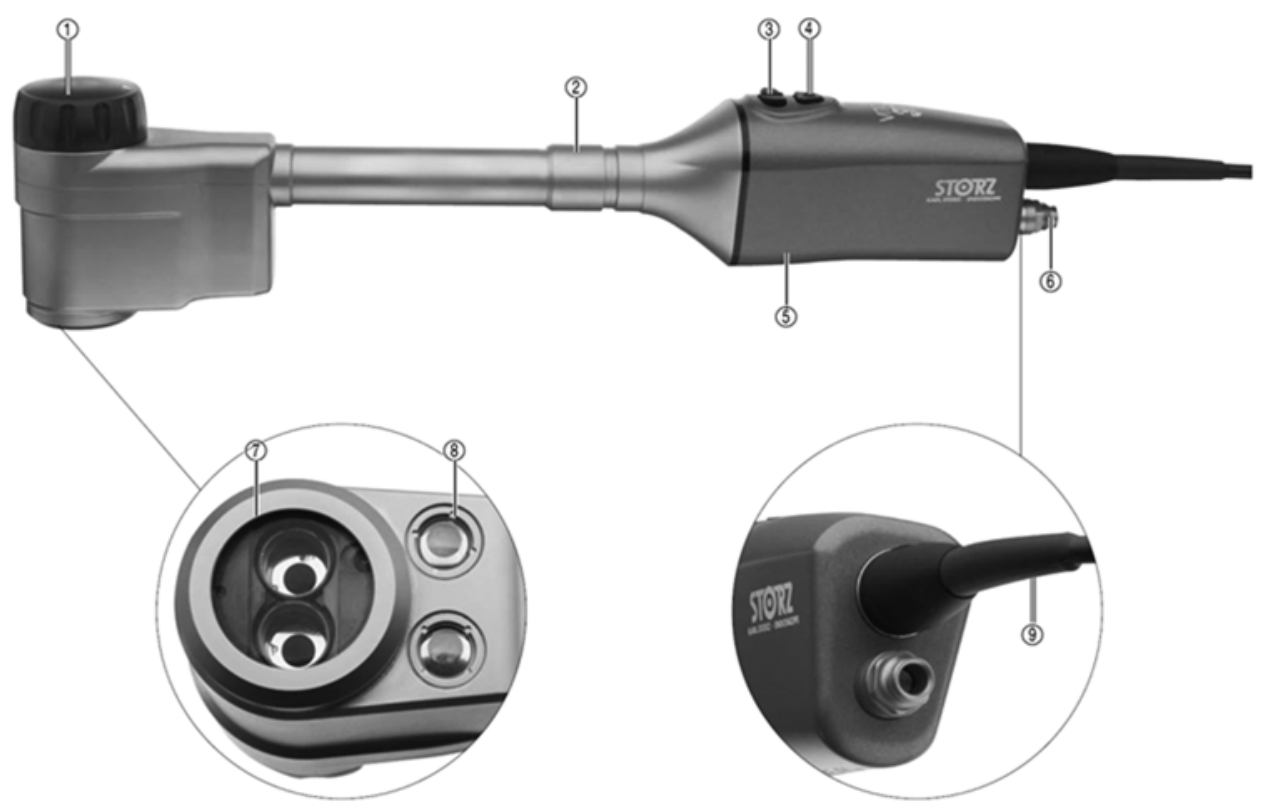

FIG. 1. VITOM 3D TH200 $90^{\circ}$ exoscope with rotary knob for horizontal image adjustment (1), reinforcement for the clamping jaw (2), rocker switch and control button (3 and 4) on the handle (5), connection to a fiber-optic light cable (6), stereo lens (7), integrated illumination unit with light outlet (8), and video connection cable (9). Copyright KARL STORZ SE \& Co KG. Published with permission.

interest (ROI), and menu navigation, the 3D exoscope can be operated by a 3D wheel-equipped IMAGE1 PILOT control unit mounted on a rotation socket and articulation stand (VERSACRANE holding arm); the intraoperative image is displayed on two 32-inch 4K 3D monitors (TM350). All these devices are from KARL STORZ SE \& Co. KG.

A brief comparison of the main baseline features of the VITOM 3D and the OM used in the control group is displayed in Table 1.

In all procedures, the VITOM 3D exoscope was connected to the holding arm via a clamping jaw and a mobile stand was used to mount the holding arm and place it close to the surgical table diagonally on the side opposite the surgeon, similar to the setup for the OM. After preparation and placement of the drape, the 3D exoscope was fitted with a sterile cover and placed about $200-500 \mathrm{~mm}$ above the surgical situs. The IMAGE1 PILOT control unit was connected to the surgical table using an articulation arm and then sterilely covered. The main monitor was positioned about 1.5 to $2.0 \mathrm{~m}$ from the consultant surgeon, on the opposite side of the surgical table, behind and next to the assisting resident. A second monitor was linked via Wi-Fi and placed opposite the main monitor (Fig. 2), be-

TABLE 1. Comparison between VITOM 3D and OM

\begin{tabular}{|c|c|c|}
\hline Characteristic & VITOM 3D & $\mathrm{OM}$ \\
\hline Focal length, $\mathrm{mm}$ & $200-500$ & $207-470$ \\
\hline Magnification, $x$ & 8-30,@ 300-mm WD & 1.2-12.8, @300-mm WD \\
\hline Cost, $€$ & Approx $100,000-200,000$ & Approx $200,000-400,000$ \\
\hline Depth of field, mm & $35-100$ & $<15$ \\
\hline Field of view, mm & $\begin{array}{l}\text { 14-145, @ 200- to 500-mm WD \& } \\
\text { depending on zoom settings }\end{array}$ & $\begin{array}{l}\text { 16.5-180, @ 207- to 470-mm WD } \\
\quad \& w / \times 10 \text { eyepiece }\end{array}$ \\
\hline Stereopsis & $3 \mathrm{D}$ & Yes \\
\hline Illumination & LED & Xenon \\
\hline Image capture & $\begin{array}{l}\text { 3D } 4 \mathrm{~K} \text { monitor }(3840 \times 2160 \\
\text { pixels })\end{array}$ & $\begin{array}{l}\text { Full HD LCD display }(1024 \times 768 \\
\text { pixels) }\end{array}$ \\
\hline Hydraulic counterbalance system & No & Yes \\
\hline Occupied space & Little & Large \\
\hline Weight, kg & 0.95 & 280 \\
\hline
\end{tabular}

Approx = approximately; HD = high-definition; LCD = liquid crystal display; LED = light-emitting diode; WD

$=$ working distance. 

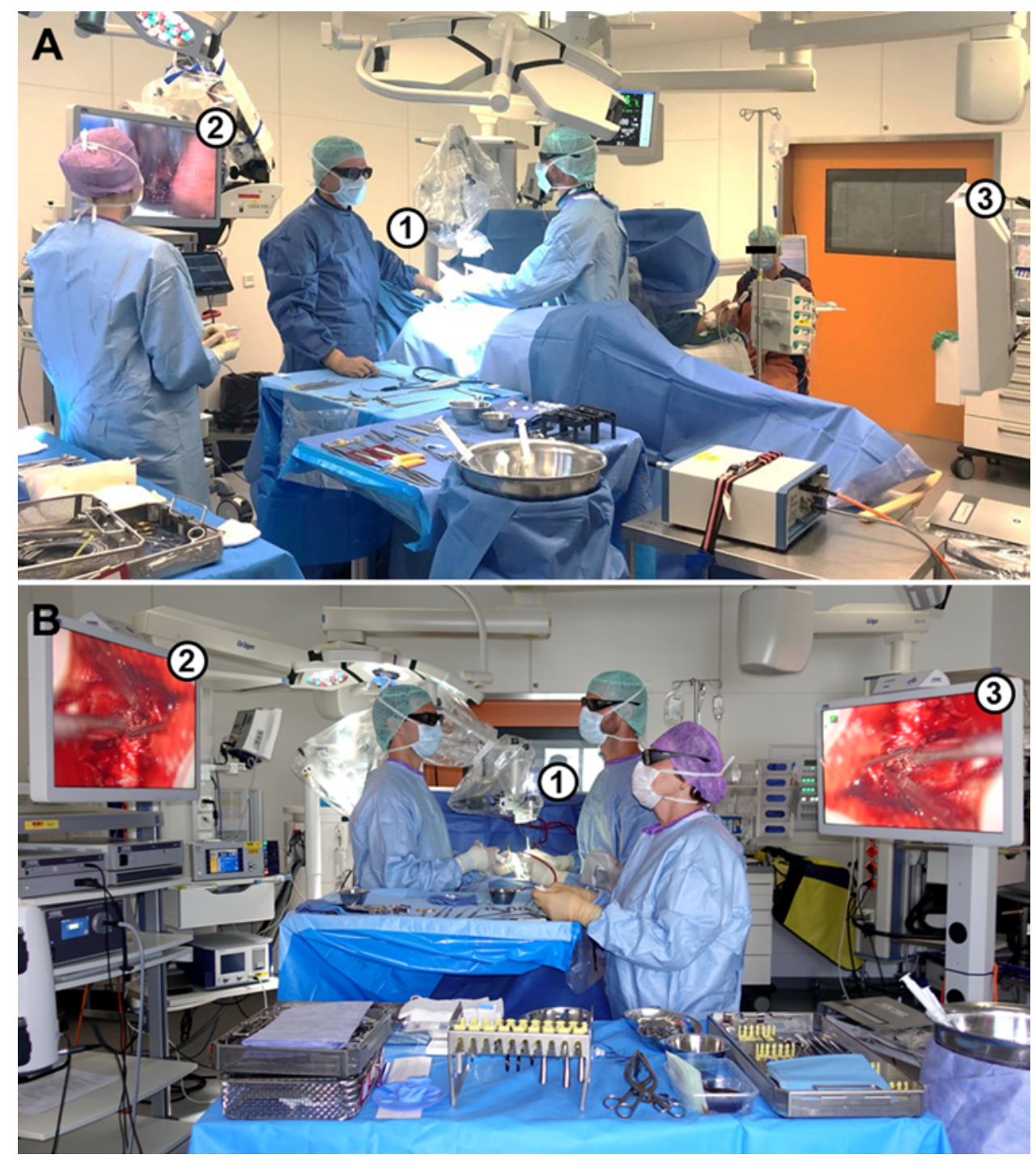

FIG. 2. Intraoperative setup in an anterior cervical $(\mathbf{A})$ and posterior lumbar $(\mathbf{B})$ spine procedure. The exoscope is positioned about $300 \mathrm{~mm}$ above the surgical field (1). The 4K 3D monitors (2 and 3) are placed using a sight-line strategy about 1.5-2.0 m opposite the surgical team. Figure is available in color online only.

hind and next to the surgeon to maintain a comfortable sight-line strategy also for the assistant. The surgical nurse stood on the left/right side of the surgeon with a direct view of the main monitor (similar to the setup of OMbased procedures with a direct view of a 2D monitor, duplicating the $\mathrm{OM}$ view). The surgical team and all staff in the OR (including anesthetists and medical students) wore polarized glasses during the procedure for $3 \mathrm{D}$ visualization. Examples of the intraoperative setups for both ACDF and LPD are given in Fig. 2A and B.

\section{Surgical Procedures}

For ACDF, patients were positioned supine and a SmithRobinson approach ${ }^{16}$ was used. The intervertebral disc, posterior longitudinal ligament, and osteophytes were resected with careful endplate preparation. Uncoforaminotomy was performed for the neuroforaminal decompression. Titanium or polyetheretherketone cages, filled with autologous bone obtained from osteophytic resection, were positioned into the intervertebral space under fluoroscopic control.

For LPD, a longitudinal midline incision was made, with exposure of the superior and inferior aspects of the laminae and involved facet joint. Then, a high-speed drill was used to create a keyhole foraminotomy, preserving at least $50 \%$ of the facet joint to maintain stability. The ligamentum flavum was resected, and the outgoing nerve root was circumferentially decompressed. In cases of stenosis, the spinal canal was widened, and the dural sac was circumferentially decompressed with Kerrison rongeurs using an "undercutting" procedure. In cases of sequestrated disc material, the material was removed with microforceps.

\section{Statistical Analysis}

Statistical patient matching was done with the aid 
TABLE 2. Baseline characteristics at index surgery

\begin{tabular}{lccc}
\hline \multicolumn{1}{c}{ Characteristic } & VITOM 3D Group & OM Group & p Value \\
\hline ACDF procedures & & & \\
\hline Sex, male/female & $13: 7$ & $13: 7$ & 1.000 \\
\hline Mean age at surgery, yrs & $57.5 \pm 10.8$ & $56.7 \pm 17.9$ & 0.859 \\
\hline Mean BMI, kg/m & $27.5 \pm 5.3$ & $26.1 \pm 4.6$ & 0.376 \\
\hline Median duration of symptoms, mos (range) & $6.0(1-132)$ & $5.8(1-96)$ & 0.654 \\
\hline Preoperative symptoms, no. (\%) & & & \\
\hline Pain & $16(80.0)$ & $19(95.0)$ & 1.000 \\
\hline Paresis & $5(25.0)$ & $10(50.0)$ & 0.303 \\
\hline Sensory disturbances & $19(95.0)$ & $17(85.0)$ & 1.000 \\
\hline Vegetative disorders & $2(10.0)$ & $2(10.0)$ & 1.000 \\
\hline Comorbidities, no. (\%) & & & \\
\hline Cardiovascular diseases & $5(25.0)$ & $6(30.0)$ & 1.000 \\
\hline Diabetes mellitus & $1(5.0)$ & $2(10.0)$ & 1.000 \\
\hline History of smoking & $9(45.0)$ & $3(15.0)$ & 0.074 \\
\hline LPD procedures & & & \\
\hline Sex, male/female & $14: 26$ & $16: 24$ & 0.946 \\
\hline Mean age at surgery, yrs & $61.9 \pm 15.0$ & $59.1 \pm 15.2$ & 0.408 \\
\hline Mean BMl, kg/m ${ }^{2}$ & $26.4 \pm 4.2$ & $26.1 \pm 4.2$ & 0.759 \\
\hline Median duration of symptoms, mos (range) & $4.3(1-59)$ & $3.8(1-48)$ & 0.623 \\
\hline Preoperative symptoms, no. (\%) & & & \\
\hline Pain & $40(100.0)$ & $39(97.5)$ & 1.000 \\
\hline Paresis & $14(35.0)$ & $25(62.5)$ & 0.864 \\
\hline Sensory disturbances & $24(60.0)$ & $20(50.0)$ & 0.333 \\
\hline Vegetative disorders & $2(5.0)$ & $2(5.0)$ & 1.000 \\
\hline Comorbidities, no. (\%) & $6(15.0)$ & $3(7.5)$ & 1.000 \\
\hline Cardiovascular diseases & & & \\
\hline Diabetes mellitus & & & \\
\hline History of smoking & & & \\
\hline
\end{tabular}

Mean values are presented \pm SD.

of propensity score matching in SPSS v3.0.4 (Felix Thoemmes [2012], arXiv: 1201.6385, Cornell University) using the nearest-neighbor-matching mode with the covariates age, sex, BMI, and surgical procedure. Statistical analysis was performed using Sigma Plot for Windows v11 (Systat Software Inc.). Differences were statistically significant if the $p$ value was $<0.05$. For comparison of groups for differences, the Student t-test was used for numeric values, the Mann-Whitney rank-sum test was used for ordinal variables, and the chi-square test was used for nominal variables, except in cases of small group comparisons, when Fisher's exact test was used. Correlation was analyzed using the Pearson product-moment correlation for continuous variables and the Spearman rank-order correlation for categorical variables.

\section{Results}

\section{Patient and Surgical Characteristics}

Of 20 patients scheduled for single-level ACDF procedures with the $3 \mathrm{D}$ exoscope, 10 patients were suffering from degenerative cervical spinal canal stenosis and 10 patients from disc herniation. Of 40 patients scheduled for single-level LPD procedures with use of the 3D exoscope, 22 patients suffered from degenerative lumbar spinal canal stenosis, and 18 patients had a mediolateral disc herniation. Due to intraoperative findings, 1 patient in the LPD group (reason: adjacent-segment stenosis) and 1 patient of the ACDF group (reason: adjacent-segment instability) initially scheduled for a single-level procedure ended up undergoing a 2-level procedure. An equal number of age-, sex-, BMI-, and procedure-matched patients undergoing OM-based ACDF and LPD procedures served as control groups.

The baseline characteristics of the ACDF and LPD cohorts are displayed in Table 2. According to our aforementioned matching criteria, there were no significant differences between the test and the respective control group.

Levels C4-5 and C5-6 (75\% of the cases) were the main location for 3D exoscope-based ACDF procedures and levels L4-5 and L5-S1 for LPD procedures (80\% of the cases), with analog location-related distribution of the 
TABLE 3. Surgical characteristics

\begin{tabular}{lccc}
\hline \multicolumn{1}{c}{ Characteristic } & $\begin{array}{c}\text { VITOM 3D } \\
\text { Group }\end{array}$ & OM Group & $\begin{array}{c}p \\
\text { Value }\end{array}$ \\
\hline ACDF procedures & & & \\
\hline Mean operative time, mins & $132 \pm 25$ & $116 \pm 37$ & 0.132 \\
\hline Mean intraoperative blood loss, $\mathrm{ml}$ & $97 \pm 83$ & $93 \pm 67$ & 0.868 \\
\hline Mean length of inpatient stay, days & $5.9 \pm 2.6$ & $7.3 \pm 5.8$ & 0.332 \\
\hline LPD procedures & & & \\
\hline Mean operative time, mins & $112 \pm 33$ & $113 \pm 37$ & 0.814 \\
\hline Mean intraoperative blood loss, $\mathrm{ml}$ & $155 \pm 218$ & $109 \pm 97$ & 0.224 \\
\hline Mean length of inpatient stay, days & $6.5 \pm 1.8$ & $7.4 \pm 7.3$ & 0.451 \\
\hline
\end{tabular}

Mean values are presented \pm SD.

surgical levels in the respective OM-based control groups ( $p=0.786$ for $\mathrm{ACDF}$ procedures; $\mathrm{p}=0.335$ for LPD procedures).

Surgical characteristics of the ACDF and LPD cohorts are displayed in Table 3. There were no significant differences between the test and the respective control group.

The mean operative time and intraoperative blood loss did not significantly differ between the first and the last quartile of procedures in the test group $(\mathrm{p}=0.564$ and $\mathrm{p}=$ 0.102 , respectively).

\section{Postoperative Outcome and Complication Rates}

Clinical outcomes for the ACDF and LPD cohorts are displayed in Table 4 and showed significant improvements of PROM parameters in both the 3D exoscope and OM control groups after 3 months compared with the preoperative status. There were no significant differences in the extent of postoperative PROM improvement between 3D exoscope- and OM-based ACDF procedures; however, in LPD procedures, postoperative PROM improvement for both back and radicular pain was slightly, but significantly, more distinct in the 3D exoscope group when compared with the OM control group.

For ACDF and LPD procedures, the patients' postoperative general clinical outcome (Odom's criteria) was graded excellent or at least good in more than $90 \%$ of the patients in both the respective 3D exoscope and OM control groups. No instances of postoperatively new/worsened sensorimotor deficits or bladder/bowel dysfunction were recorded, and the patients' general performance in daily life was unaffected (median Barthel Index of 100\%) in either group.

\section{Questionnaire Rating for 3D Exoscope Application}

The median values of the surgeons', assistants', and OR nursing staff's questionnaire ratings for 3D exoscopebased ACDF and LPD procedures in comparison with the OM are displayed in Table 5; examples for 3D exoscopeand OM-based intraoperative visualization are given in Fig. 3.

According to the attending surgeon and assisting resident, the intraoperative handling of instruments during 3D exoscope procedures was rated to be similar to that of the OM, while the intraoperative handling/reposition of the 3D exoscope and the adjustment of magnification/ focal length via the control unit were rated to be comparable, but slightly worse, compared with the OM usability. Depth perception, image quality, and illumination during 3D exoscope-based ACDF and LPD procedures were judged to be "good" but slightly inferior to the OM quality, which was especially emphasized by the surgical team during ACDF procedures with long approaches in obese patients; conversion from the 3D exoscope to the $\mathrm{OM}$ was done by the attending surgeon in 1 LPD procedure to optimize image quality during a sequestrectomy and in 2 ACDF procedures to improve depth perception and illumination during the anterior approach. The comfort level of the intraoperative posture was rated to be superior by the surgical team in all 3D exoscope procedures, which was especially pointed out during undercutting maneuvers in LPD procedures.

For the OR nursing staff participating in 3D exoscope procedures, the intraoperative storage of the sterile $3 \mathrm{D}$ exoscope was rated to be slightly inferior, while the sterile covering itself and the ability to follow the consecutive procedural steps and appropriately assist the surgeon were rated to be superior compared with the OM (especially for ACDF procedures). The mean time of exposure for the sterile covering of the $3 \mathrm{D}$ exoscope was significantly lower than that of the OM $(81 \pm 33$ vs $111 \pm 27$ seconds, respectively; $\mathrm{p}<0.001$ ).

In no case was wearing of $3 \mathrm{D}$ glasses rated to be disruptive, and there were no complaints of headaches, dizziness, or nausea due to the intraoperative application of the 3D exoscope.

\section{Discussion}

Since the late 1990s, promising efforts to replace the OM with a 3D exoscope have been attempted in many medical disciplines, including, for example, otolaryngology and reconstructive surgery. ${ }^{17,18}$ Comparable attempts have been made for spinal microsurgery in recent years. The cumulative evidence for the feasibility of 3D exoscope-based spinal microsurgery, however, is still very limited, and the current literature mainly consists of small and mostly uncontrolled case reports with a dominance in the use of the STORZ VITOM systems. ${ }^{4-10}$ However, systematic studies comparing a 3D exoscopic system with other devices for visualization and magnification, such as the $\mathrm{OM}$, are still missing in spine surgery.

Here we prospectively compare the utilization of a state-of-the-art 4K 3D exoscope with a conventional OM in cases of degenerative spine surgeries; to systematically evaluate whether the 3D exoscope could be an alternative to the $\mathrm{OM}$, we specifically focused on narrowly defined standard procedures of the cervical and lumbar spine (ACDF and LPD).

\section{The 3D Exoscope Compared With the Operating Microscope and Endoscope in Degenerative Spine Surgeries}

In our study, surgical outcomes after use of the 3D exoscope were comparable to those obtained with the OM. We did not find any significant differences in operative time, 
TABLE 4. Outcome parameters during follow-up*

\begin{tabular}{|c|c|c|c|c|c|c|c|}
\hline \multirow[b]{2}{*}{ Characteristic } & \multicolumn{3}{|c|}{ VITOM 3D Group } & \multicolumn{3}{|c|}{ OM Group } & \multirow[b]{2}{*}{ p Value } \\
\hline & Preop & Postop & $p$ Value & Preop & Postop & $p$ Value & \\
\hline \multicolumn{8}{|c|}{ ACDF procedures (range) } \\
\hline Median VAS-N & $5(0-8)$ & $0(0-4)$ & $<0.001$ & $5(0-8)$ & $0(0-1)$ & $<0.001$ & 0.744 \\
\hline Median VAS-R & $5(0-8)$ & $0(0-3)$ & $<0.001$ & $5(0-8)$ & $0(0-3)$ & $<0.001$ & 0.932 \\
\hline Median NDI & $2(1-3)$ & $1(1-3)$ & 0.001 & $2(1-3)$ & $1(1-3)$ & $<0.001$ & 0.052 \\
\hline \multicolumn{8}{|c|}{ LPD procedures (range) } \\
\hline Median VAS-B & $7(0-9)$ & $0(0-9)$ & $<0.001$ & $6(0-8)$ & $2(0-7)$ & $<0.001$ & 0.023 \\
\hline Median VAS-R & $7(0-9)$ & $0(0-7)$ & $<0.001$ & $6(0-8)$ & $1(0-5)$ & $<0.001$ & 0.044 \\
\hline Median ODI & $2(1-3)$ & $1(1-3)$ & $<0.001$ & $2(1-3)$ & $1(1-2)$ & $<0.001$ & 0.535 \\
\hline
\end{tabular}

VAS = visual analog scale; VAS-B = VAS for back pain; VAS-N = VAS for neck pain; VAS-R = VAS for radicular pain.

* NDI scoring system: grade 1 (no disability: 0\%-8\%); grade 2 (mild disability: 10\%-28\%); grade 3 (moderate disability: $30 \%-48 \%$ ); grade 4 (severe disability: 50\%-68\%); and grade 5 (complete disability: > 68\%). ODI scoring system: grade 1 (minimal disability: $0 \%-20 \%$ ); grade 2 (moderate disability: $21 \%-40 \%$ ); grade 3 (severe disability: $41 \%-60 \%$ ); grade 4 (crippling back pain: $61 \%-80 \%$ ); and grade 5 (complete disability: > 80\%).

$\dagger$ Each $p$ value reflects the change in scores between the VITOM 3D and OM groups.

intraoperative blood loss, complications, and postoperative length of inpatient stay between patients treated with the 3D exoscope and those treated with the OM. Similar experiences were seen in previous case series. ${ }^{4,5,10}$ Moreover, we could additionally show that postoperative pain reduction and functional improvement in NDI/ODI scores were equally effective in 3D exoscopic-based procedures compared to the standard OM-based procedures.

It is known that new visualization devices and operative techniques like endoscopy for lumbar discectomy are accompanied by a learning curve and, initially, an increased rate of complications. ${ }^{19}$ In the case of a 3D exoscope, however, the visualization situation is more similar to that of the OM because the exoscope is placed outside the body cavity and has a similar focal distance, while endoscopes exhibit a rather short focal distance of approximately 3-20 $\mathrm{mm}$, which easily can result in surgical instruments hitting the optics or obstructing visualization during the procedure. The larger field of view and working distance of a 3D exoscope allow for the positioning of traditional spinal operative tools and contribute to a minimal learning curve since surgeons do not have to adapt to or customize new surgical instruments/techniques. After a 2-week period of training in the use of the intraoperative 3D exoscope, we could not find any significant differences in surgical outcomes (e.g., operative time or intraoperative blood loss) between the first and the last quartile of study-relevant procedures. Similar experiences in terms of a minimal learning curve with 3D exoscopes were discussed in previous case series. ${ }^{4}$ In addition, with the lens of the 3D exoscope positioned outside the body cavity, the problems typically faced during endoscopy-e.g., repetitive cleaning of fogged endoscopic lenses-can be avoided. Lastly, problems of lateral image inversion are prevented because of the ability of the $3 \mathrm{D}$ camera head to be rotated $360^{\circ}$ to match the resulting image and surgeon's position in relation to the surgical situs.

When using two Wi-Fi-connected 4K 3D monitors placed at opposite sides of the operating and assisting surgeons, the intraoperative setup of 3D exoscopic procedures is similar to the setup during operations with an $\mathrm{OM}$, and there is no need for changes in the habitual positioning of surgeons/OR nurses, even though the intraoperative positioning of the two-monitor-equipped 3D exoscope system can be challenging in the OR, which, in our series, was additionally equipped with an OM. Visualization properties of the 3D exoscope with regard to depth perception, image quality, and illumination were rated in median as comparable to those of the $\mathrm{OM}$ (with minor inferiority particularly in ACDF procedures with long approaches) by the surgical team; this is in line with previous case series and cadaveric laboratory experiences ${ }^{4-10,20}$ Although nonsurgical OR staff is an essential factor for the smooth intraoperative workflow and the success of surgery, none of the previous case series have evaluated the rating of nonsurgical staff participating in 3D exoscopic procedures; we aimed to close this information gap and showed that visualization of the intraoperative process flow and surgical situs in 3D exoscopic procedures was rated to be comparable to and, in ACDF procedures, even slightly superior to OM-based procedures.

While producing similar surgical/clinical outcomes with satisfying visualization properties, the 3D exoscope is smaller and lighter (obviating the need for a heavy counterweight system) and can be transported more easily than the $\mathrm{OM}$; beyond that, the intraoperative sterile covering is faster and easier. Moreover, due to its large dimensions, the $\mathrm{OM}$ is usually positioned after finishing the dissection of the spine, while in our experience the 3D exoscope was regularly placed during the initial dissection, mostly because it does not restrict the surgeon's direct view of the surgical sites. Wearing polarized 3D glasses was never rated as disruptive in our study, and there was no hint of a headache, eye strain, or nasal pain induced by 3D exoscopic procedures in the participating OR staff (whether a permanent spectacle wearer or not). The 3D exoscope offers the unique advantage of facilitating a 3D visualization in the same high quality for all persons present in the OR and may, therefore, have an important role in educating and training all people present in the OR-for example, with 


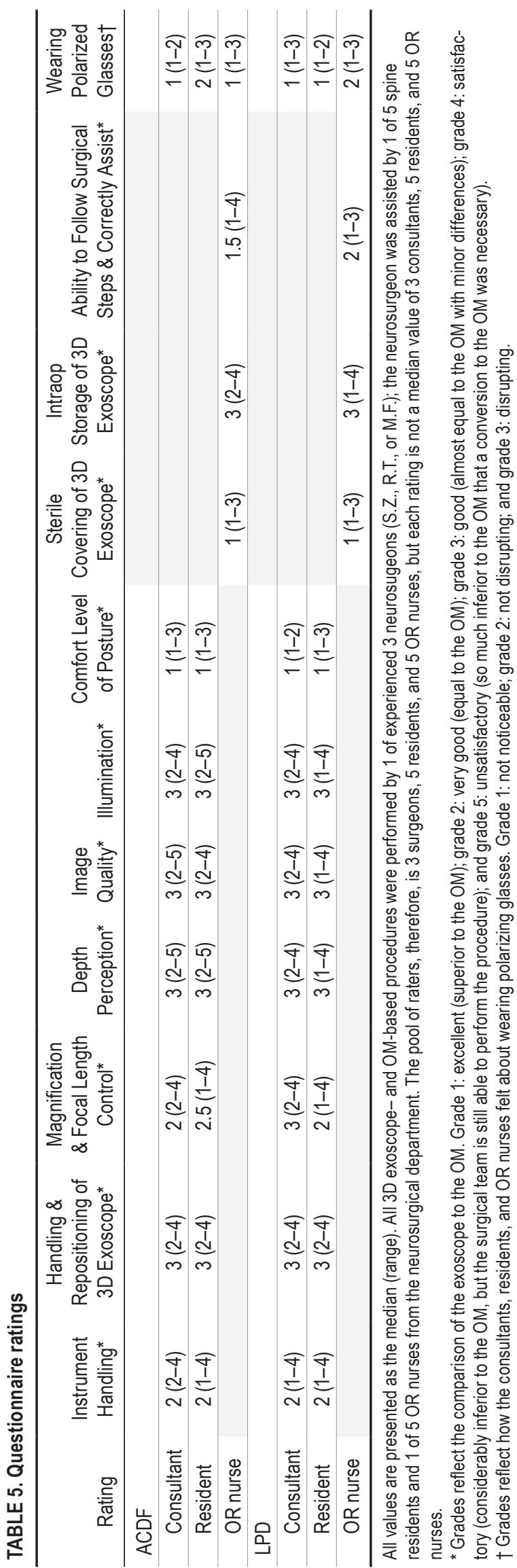

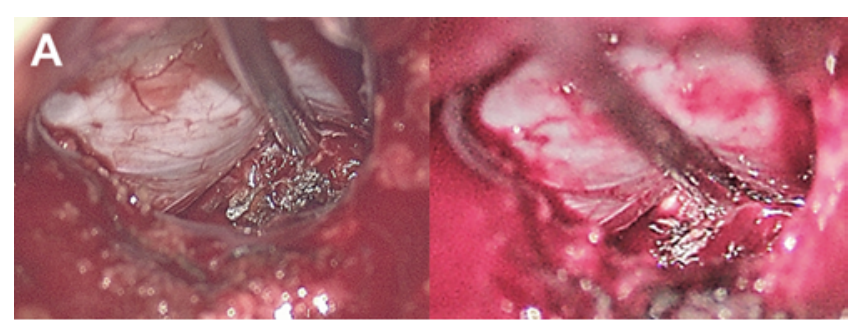

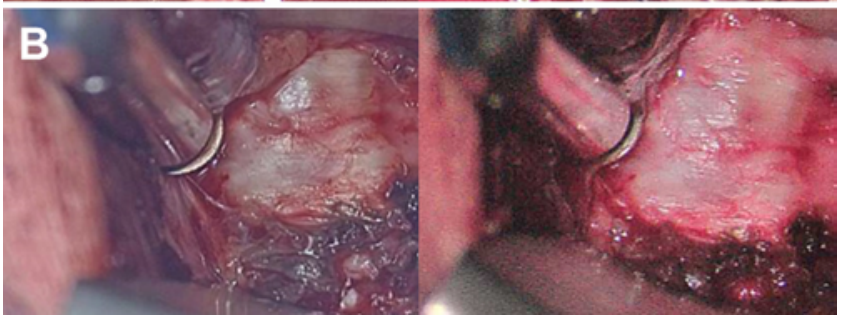

FIG. 3. Intraoperative visualization of a posterior lumbar spine procedure with the exposed dural sac held aside by the surgical aspirator to allow a view of the posterior longitudinal ligament $(\mathbf{A})$ and of an anterior cervical spine procedure after insertion of a retractor pin to allow a view of the anterior longitudinal ligament (B) with the VITOM 3D exoscope (left) and the OM (right). Figure is available in color online only.

anatomical structures being understood and studied more intuitively with the real-time 3D view on a $4 \mathrm{~K}$ screen.

A unique benefit of the 3D exoscope is the significantly improved ergonomics and body mechanics for the surgical team, which were reported by both the operating and assisting surgeons in nearly all spine procedures performed during our study period and are in line with previous case series and cadaveric laboratory experiences. ${ }^{4-10,20}$ When using the OM, surgeons are often required to lean forward or to bend their neck for prolonged periods in order to look into the eyepieces, while maintaining this uncomfortable position to ensure sufficient maneuverability for surgical instruments. This is especially true for minimally invasive procedures requiring small incisions and the need to explore and dissect larger areas or cavities through minimized approaches. The high maneuverability of the 3D exoscope and the innovative renunciation from the faceto-machine interface, however, allow the 3D exoscopeassisted surgical team to work in a natural and comfortably straight upright ergonomic posture during the entire procedure, minimizing the surgeons' stress and operative fatigue. The large field of view and the long working distance allow manipulation of the 3D exoscope without the constraints of bony anatomy.

An economic advantage of 3D exoscopes is its lower cost compared with an OM; the estimated cost for the exoscopic setup used in our study is approximately $€ 70,000$. Parts of the equipment (e.g., the monitors) can be utilized in other surgical procedures (e.g., endoscopy) or specialties. Moreover, since digital cameras, monitors, and light sources are mass products that are utilized in a variety of industrial sectors and are steadily improved, it is supposed that the costs of digital visualization technologies will steadily decrease, while the highly specialized and elaborate production of OMs will likely remain a costly niche industrial branch. With a lower cost and smaller size, the $3 \mathrm{D}$ exoscope is already now a viable and economical 
alternative to the OM for smaller hospitals, outpatient surgical centers or self-employed surgeons, and mobile surgical units. The last point might take on an important role, especially in developing countries.

\section{Limitations of the 3D Exoscope in Degenerative Spine Surgeries}

Although the visualization properties of the 3D exoscope with regard to depth perception, image quality, and illumination were rated in median as comparable to those of the OM by the surgical team, a minor inferiority-especially for depth perception and illumination in ACDF with long approaches in very obese patients-was seen in our study; in 3 cases, conversion from using the 3D exoscope to employing the $\mathrm{OM}$ was performed by the attending surgeon to optimize visualization-although we want to state that conversion was a subjective preference of the attending surgeon and not by necessity to protect the patient from harm. The subjective better depth perception when using the $\mathrm{OM}$ was also the most frequent reason for the attending and resident surgeons to prefer the $\mathrm{OM}$ when performing future spine procedures in our study. Although most smaller and uncontrolled case series of 3D exoscopebased spine surgery unreflectingly rate the visualization properties of 3D exocopes as "outstanding," "excellent," or "superior" due to the mere subjective estimation of the contributing authors without describing the criteria of evaluation, ${ }^{4,89}$ the only comprehensible comparison of 3D exoscope- and OM-based spine procedures available in the literature, a report by Oertel and Burkhardt, ${ }^{5}$ shows hints at the inferiority of the 3D exoscope with regard to depth perception/image quality in certain circumstances, which is in line with the results of our large controlled prospective trial.

Another limitation of the 3D exoscope concerns the ease of repositioning the holding arm. Handling and repositioning of the 3D exoscope were rated in median to be comparable but slightly inferior to the OM, and we often found that the mechanics of the scope holder were not as easy to reposition as the hydraulic counterbalance system of the OM, which is in line with the findings in previous smaller case series., ${ }^{4,9,2,21,22}$ Even though the actual control unit of the VITOM 3D system partially facilitates repositioning and shifting the focus of the 3D camera a few millimeters without repositioning the scope holder, the need to refocus/reposition the 3D exoscope frequently during surgical interventions without losing sight of the surgical field remains unaltered. A hydraulic counterbalance-for example, a robotic mechanism for the scope holder-could be the solution to enable an easier and more precise repositioning of the 3D exoscope and improve its functionality.

\section{Conclusions}

When comparing a state-of-the-art 4K 3D exoscope with a conventional $\mathrm{OM}$ in standard cervical and lumbar spinal surgeries, we were able to demonstrate the safety and efficacy of 3D exoscope-based procedures with the unique advantage of excellent comfort and ergonomy for the surgical team, resulting in comparable clinical outcomes for our patients. The 3D exoscope should be con- sidered a practical and economical alternative for smaller hospitals, outpatient clinics, and mobile surgical units. However, the drawbacks of slightly decreased quality of visualization/illumination and reduced ease of handling and repositioning of the 3D exoscope must be kept in mind, particularly in more complex spine or spinal cord procedures.

\section{Acknowledgments}

We are grateful to all patients participating in the study. Special thanks are directed to KARL STORZ SE \& Co. KG, Tuttlingen, Germany, for providing the exoscopic equipment.

\section{References}

1. Jacobson JH II, Wallman LJ, Schumacher GA, et al. Microsurgery as an aid to middle cerebral artery endarterectomy. $J$ Neurosurg. 1962;19:108-115.

2. Caspar W. A new surgical procedure for lumbar disk herniation causing less tissue damage through a microsurgical approach. Adv Neurosurg. 1977;4:74-77.

3. Yaşargil MG. Microsurgical operation of herniated lumbar disc. In: Wüllenweber R, Brock M, Hamer J, et al., eds. Lumbar Disc Adult Hydrocephalus. Springer; 1977:81. Advances in Neurosurgery; vol 4.

4. Shirzadi A, Mukherjee D, Drazin DG, et al. Use of the video telescope operating monitor (VITOM) as an alternative to the operating microscope in spine surgery. Spine (Phila Pa 1976). 2012;37(24):E1517-E1523.

5. Oertel JM, Burkhardt BW. Vitom-3D for exoscopic neurosurgery: initial experience in cranial and spinal procedures. World Neurosurg. 2017;105:153-162.

6. Barbagallo GMV, Certo F. Three-dimensional, high-definition exoscopic anterior cervical discectomy and fusion: a valid alternative to microscope-assisted surgery. World Neurosurg. 2019;130:e244-e250.

7. Ricciardi L, Chaichana KL, Cardia A, et al. The exoscope in neurosurgery: an innovative "point of view". A systematic review of the technical, surgical and educational aspects. World Neurosurg. 2019;124:136-144.

8. Beez T, Munoz-Bendix C, Beseoglu K, et al. First clinical applications of a high-definition three-dimensional exoscope in pediatric neurosurgery. Cureus. 2018;10(1):e2108.

9. Krishnan KG, Schöller K, Uhl E. Application of a compact high-definition exoscope for illumination and magnification in high-precision surgical procedures. World Neurosurg. 2017;97:652-660.

10. Kwan K, Schneider JR, Du V, et al. Lessons learned using a high-definition 3-dimensional exoscope for spinal surgery. Oper Neurosurg (Hagerstown). 2019;16(5):619-625.

11. Langer DJ, White TG, Schulder M, et al. Advances in intraoperative optics: a brief review of current exoscope platforms. Oper Neurosurg (Hagerstown). 2020;19(1):84-93.

12. Vernon H, Mior S. The Neck Disability Index: a study of reliability and validity. J Manipulative Physiol Ther. 1991;14(7):409-415.

13. Fairbank JC, Couper J, Davies JB, O'Brien JP. The Oswestry low back pain disability questionnaire. Physiotherapy. 1980;66(8):271-273.

14. Mahoney FI, Barthel DW. Functional evaluation: the Barthel Index. Md State Med J. 1965;14:61-65.

15. Odom GL, Finney W, Woodhall B. Cervical disk lesions. $J$ Am Med Assoc. 1958;166(1):23-28.

16. Smith GW, Robinson RA. The treatment of certain cervicalspine disorders by anterior removal of the intervertebral disc and interbody fusion. J Bone Joint Surg Am. 1958;40A(3):607-624. 
17. Crosetti E, Arrigoni G, Manca A, et al. 3D exoscopic surgery (3Des) for transoral oropharyngectomy. Front Oncol. 2020;10:16.

18. Piatkowski AA, Keuter XHA, Schols RM, van der Hulst RRWJ. Potential of performing a microvascular free flap reconstruction using solely a $3 \mathrm{D}$ exoscope instead of a conventional microscope. J Plast Reconstr Aesthet Surg. 2018;71(11):1664-1678.

19. Wang B, Lü G, Patel AA, et al. An evaluation of the learning curve for a complex surgical technique: the full endoscopic interlaminar approach for lumbar disc herniations. Spine J. 2011:11(2):122-130.

20. Herlan S, Marquardt JS, Hirt B, et al. 3D exoscope system in neurosurgery-comparison of a standard operating microscope with a new 3D exoscope in the cadaver lab. Oper Neurosurg (Hagerstown). 2019;17(5):518-524.

21. Rossini Z, Cardia A, Milani D, et al. VITOM 3D: preliminary experience in cranial surgery. World Neurosurg. 2017;107:663-668.

22. Mamelak AN, Nobuto T, Berci G. Initial clinical experience with a high-definition exoscope system for microneurosurgery. Neurosurgery. 2010;67(2):476-483.

\section{Disclosures}

The exoscope was provided by KARL STORZ SE \& Co. KG.

The authors report no conflict of interest concerning the materials or methods used in this study or the findings specified in this paper.

\section{Author Contributions}

Conception and design: Siller, Zausinger. Acquisition of data: Siller, Zoellner, Fuetsch, Trabold, Zausinger. Analysis and interpretation of data: Siller, Zoellner, Zausinger. Drafting the article: Siller. Critically revising the article: Siller, Zausinger. Reviewed submitted version of manuscript: all authors. Approved the final version of the manuscript on behalf of all authors: Siller. Statistical analysis: Siller. Administrative/technical/material support: Siller. Study supervision: Siller.

\section{Supplemental Information}

Previous Presentations

Portions of this paper were presented orally at the Annual Spine Section Meeting of the German Society of Neurosurgery, Giessen, Germany, September 7, 2019, and at the 14th Annual Meeting of the German Spine Society, Munich, Germany, November 28, 2019.

\section{Correspondence}

Sebastian Siller: Clinic of the University of Munich (Ludwig Maximilian University), Munich, Germany. sebastian.siller@med. uni-muenchen.de. 